\title{
Comparison Of The Genesis Solar Wind Regime Algorithm Results With Solar Wind Composition Observed By ACE
}

\author{
Daniel B. Reisenfeld*, John T. Steinberg*, Bruce L. Barraclough*, Eric E. Dors*, \\ Roger C. Wiens", Marcia Neugebauer ${ }^{\dagger}$, Alysha Reinard ${ }^{\ddagger}$, and Thomas Zurbuchen \\ *Space and Atmospheric Sciences, MS-D466, Los Alamos National Laboratory, Los Alamos, NM 87544 \\ ${ }^{\prime}$ Lunar and Planetary Laboratory, Bldg. 92, University of Arizona, Tucson, AZ 85721 \\ Department of Atmospheric, Oceanic and Space Sciences, University of Michigan, Ann Arbor, MI 48109
}

\begin{abstract}
Launched on 8 August 2001, the NASA Genesis mission is now collecting samples of the solar wind in various materials, and will return those samples to Earth in 2004 for analysis. A primary science goal of Genesis is the determination of the isotopic and elemental composition of the solar atmosphere from the solar wind material returned. In particular, Genesis will provide measurements of those species that are not provided by solar and in situ observations. We know from in situ measurements that the solar wind exhibits compositional variations across different types of solar wind flows. Therefore, Genesis exposes different collectors to solar wind originating from three flow types: coronal hole, coronal mass ejection (CME), and interstream flows. Flow types are identified using in situ measurements of solar wind protons, alphas, and electrons from electrostatic analyzers carried by Genesis. The flow regime selection algorithm and subsequent collector deployment on Genesis act autonomously. We present an assessment of composition variations of $\mathrm{O}, \mathrm{He}$, and $\mathrm{Mg}$ ions observed by ACE/SWICS concurrent with Genesis observations, and compare these to the Genesis algorithm decisions. Not only does this serve as a test of the algorithm, the compilation of composition vs. regime will be important for comparison to the abundances determined from sample analysis at the end of the mission.
\end{abstract}

\section{INTRODUCTION}

The Genesis spacecraft, launched on 8 August 2001, will be the first spacecraft ever to return from interplanetary space. Its goal is to collect samples of solar wind and return them to Earth for isotopic and elemental analysis [1]. Two instruments are used to collect samples. One is a set of panels arrayed with wafers that passively collect solar wind material. The second is the solar wind ion concentrator, which uses a set of large electrostatic elements to focus solar wind ions onto diamond and silicon carbide substrates and thereby increase the flux of ions implanted [2]. The resulting concentrated sample will be important for determining the solar wind oxygen isotope ratios to the desired accuracy.

The principal scientific rational of Genesis is to understand the composition of the solar nebula from which our solar system formed, and to use this as a baseline for comparison with present-day planetary compositions. The outer layers of the Sun are thought to be relatively unchanged since the formation of the solar nebula. The solar wind is known to be elementally fractionated relative to the solar photosphere [3], though fractionation of isotopes is likely to be relatively minor. In addition, we know from in situ measurements that the solar wind exhibits compositional variations across different types of solar wind flows [4]. Significant elemental variations are seen between fast and slow wind types. Coronal mass ejections (CMEs) exhibit yet another set of abundances. Therefore, Genesis exposes different arrays of collectors to solar wind originating from these three flow types: fast wind, slow wind, and CMEs. Flow types are identified using in situ measurements of solar wind protons, alphas, and electrons from electrostatic analyzers carried by Genesis [5]. An on-board regime selection algorithm uses these data to determine in which of the three regimes the spacecraft is currently immersed, and the appropriate collection panel is deployed [6]. The algorithm takes into account the proton speed, proton temperature, alpha particle abundance, and the 
presence of counter-streaming electrons as determined onboard.

The solar wind flow regimes serve as proxies for the solar wind abundance regimes, which are ultimately what we desire to isolate on different panels; thus, we would like to know how successfully the algorithm discriminates among abundance regimes. Furthermore, the concentrator focuses oxygen ions of different charge states differently, so we need to know the relative amounts of the different oxygen charge states collected over the mission in order to model the oxygen implantation distribution across the target. Genesis does not have instruments capable of performing time-resolved heavy-ion composition measurements; thus, to address these issues, we turn to the Solar Wind Ion Composition Spectrometer (SWICS) on board the Advanced Composition Explorer (ACE) [7] to provide composition information that we can then correlate with the Genesis data.

An equally important goal of this study is to make use of the Genesis regime selection algorithm to investigate the scientific question: "How well does speed correlate with composition?" This question has been investigated by many [8], but here we extend the analysis by addressing the following issue: Because of hydrodynamic processing of the solar wind as it propagates outward from the Sun, the solar wind speed measured at $1 \mathrm{AU}$ may be different from the flow speed as it left the inner corona, especially for plasma at flow interfaces. Originally fast wind may be slowed and vice-versa. Thus, the speed measured at $1 \mathrm{AU}$ at these interfaces may not correlate with the composition as expected. However if we consider the speed history, we can assess whether the wind has been accelerated or decelerated and then use SWICS observations to evaluate whether taking this into account improves the regime separation.

\section{REGIME SELECTION ALGORITHM}

The Genesis regime selection algorithm processes data from the Genesis electron and ion spectrometers, determining the solar wind regime in real time on board the spacecraft. The algorithm is fully described in Neugebauer et al. [6]. Here we focus on those aspects of the algorithm relevant to composition. Figure 1 shows the decision flow chart used to determine one of three cases: whether the observed solar wind is (1) fast wind originating from a coronal hole $(\mathrm{CH}),(2)$ slow wind from an interstream source (IS), or (3) CME plasma. Based on this determination, collection array changes are made within minutes of determination of a regime change.

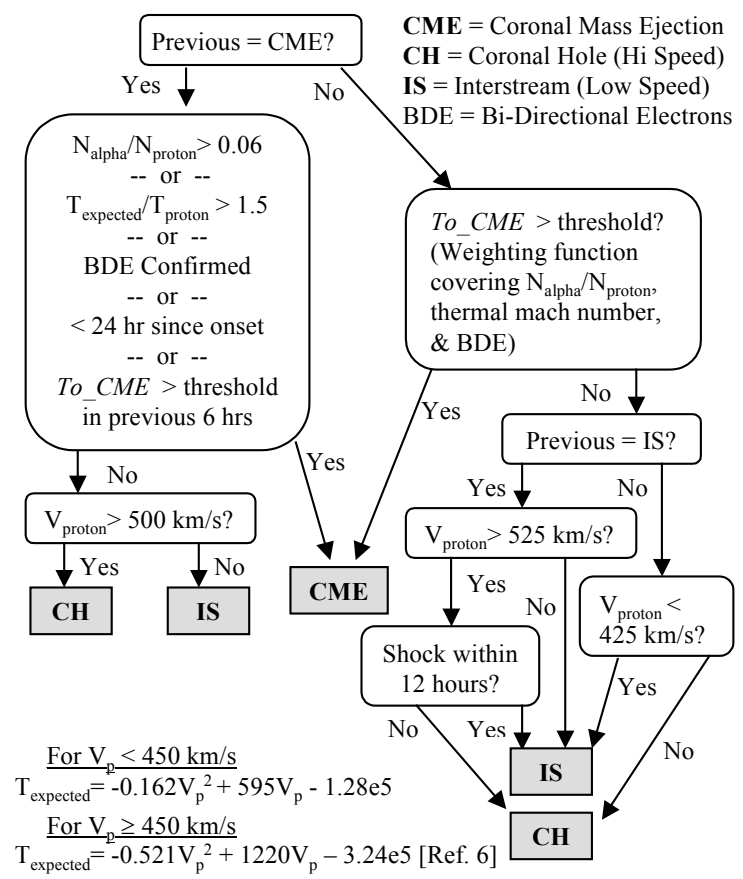

FIGURE 1. Flow chart showing the Genesis on-board logic for determining the solar wind regime in real time.

The following assumptions underlie the algorithm:

1) $\mathrm{CH}$ and IS flow (collectively referred to as quasi-stationary flow) originate from solar sources that exhibit distinct abundance signatures. Such flows are characterized by speed. We therefore identify $\mathrm{CH}$ and IS material based on solar wind speed and use the speed to determine which collector array to deploy.

2) Elemental abundances in CMEs are highly variable and are not necessarily related to $\mathrm{CH}$ or IS abundances. We therefore identify and collect CME material on a separate collector.

3) Although solar wind speed is related to the outflow source (CH or IS), the solar wind undergoes hydrodynamic evolution which can obscure the source signature. The recent history of the solar wind can be used to partially recover some of this information. In particular: (a) on the leading edge of a stream, initially slow wind has been accelerated; thus, although at the location of observation the wind is fast, it will have an abundance expected of slower wind. Thus when a slow-to-fast transition occurs, the speed set point for retracting the IS collector and deploying the $\mathrm{CH}$ collector is set relatively high: $525 \mathrm{~km} \mathrm{~s}^{-1}$. (b) On the trailing side of a fast stream, originally fast wind has been decelerated due to the rarefaction caused by fast 
wind outrunning slow wind; thus, although at the location of the observation the trailing wind is slow, it will have the abundance of faster flow. Thus when a fast-to-slow transition is observed, the speed set point for retracting the $\mathrm{CH}$ collector and deploying the IS collector is set relatively low: $425 \mathrm{~km} \mathrm{~s}^{-1}$.

\section{SOLAR WIND COMPOSITION MEASUREMENTS}

To test the performance of the regime algorithm, we considered a combination of elemental abundance ratios and charge-state ratios derived from ACE/SWICS observations coincident with Genesis operation from 20 September 2001 to 6 May 2002. For this time, the on-board algorithm determined the following fractions of time spent in each regime: fast wind (11\%), slow wind (57\%), and CME (32\%).

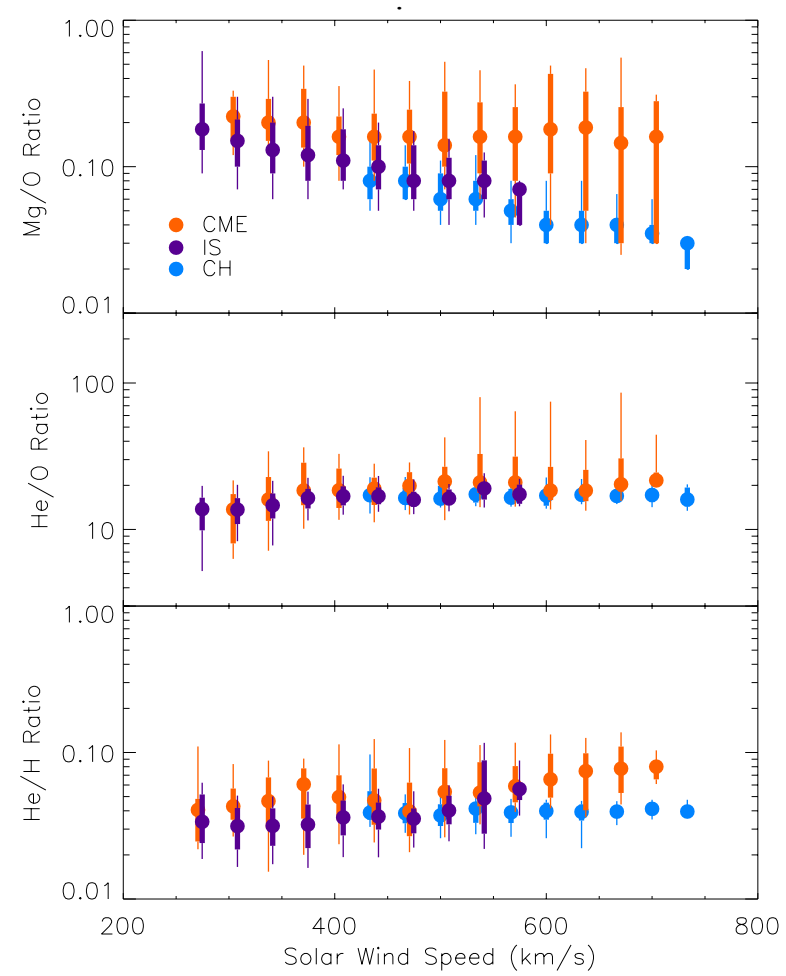

FIGURE 2. Elemental abundance ratios for $\mathrm{Mg} / \mathrm{O}, \mathrm{He} / \mathrm{O}$ and $\mathrm{He} / \mathrm{H}$ as a function of solar wind proton speed and Genesis solar wind regime. The points indicate the median values for data within bins $33 \mathrm{~km} / \mathrm{s}$ wide. The thin lines span the 10- to 90-percentile ranges, and the heavy lines span the 25- to 75-percentile ranges of the data within each bin.

Elemental fractionation of the solar wind depends on the time it takes for an element to become ionized in the chromosphere, which in turn, depends strongly on the first ionization potential (FIP). Low-FIP elements $($ FIP $<10 \mathrm{eV})$ are enhanced over their photospheric abundance relative to high-FIP elements [9]. This effect is most strongly observed in IS flow, where the enhancement factor is 4-5, whereas the factor is $\leq 2$ for $\mathrm{CH}$ flow [4]. In Figure 2 we show selected abundance ratios measured by SWICS, separated according to the three solar wind regimes as determined by Genesis, and further sorted by solar wind proton speed.

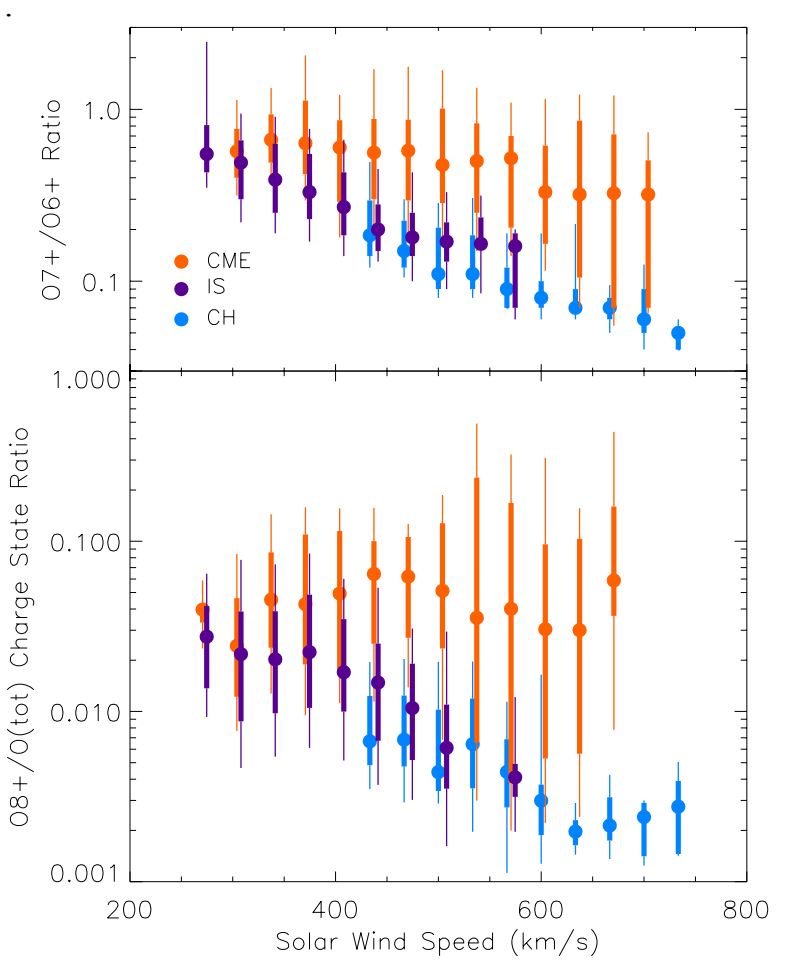

FIGURE 3. Charge state ratios for $\mathrm{O}^{7+} / \mathrm{O}^{6+}$ and $\mathrm{O}^{8+} / \mathrm{O}_{\text {total }}$ as a function of proton speed and Genesis solar wind regime. $\mathrm{O}_{\text {total }}$ is the total number of oxygen ions of all charge states.

The top panel of Figure 2 shows the ratio of magnesium (a low-FIP element) to oxygen (a high-FIP element) [10]. For the quasi-stationary wind, we see a steady monotonic decrease in the $\mathrm{Mg} / \mathrm{O}$ ratio with speed, varying across a factor of 6 . The smooth decrease of $\mathrm{Mg} / \mathrm{O}$ with speed shows us that, although it is common to refer to different FIP fractionation values for IS and $\mathrm{CH}$ flows (as we have done in the paragraph above), there is not a sharp abundance boundary between high and low speed. Rather, at least to first order, fractionation is a continuous function of proton speed. Importantly, where the IS and $\mathrm{CH}$ regimes overlap, we do see a clear separation in the $\mathrm{Mg} / \mathrm{O}$ ratio. We attribute this to the interplanetary processing at flow boundaries describe above. We 
also note that the $\mathrm{Mg} / \mathrm{O}$ ratio shows significantly less variance for the $\mathrm{CH}$ regime than for the IS regime.

In contrast, the $\mathrm{Mg} / \mathrm{O}$ ratio for $\mathrm{CME}$ flow is remarkably steady at an average value of 0.20 , equal to the ratio seen in IS flow at $\sim 300 \mathrm{~km} \mathrm{~s}^{-1}$. Although the $\mathrm{Mg} / \mathrm{O}$ average value remains constant, the distribution skews to smaller values with increasing proton speed. We attribute this to the conservative design of the algorithm: to minimize the risk that the $\mathrm{CH}$ and IS arrays are contaminated by CME material, the algorithm maintains the CME regime state for at least 18 hours, plus an additional 6 hours after CME signatures are last detected. In this way, because CME signatures are often intermittent, enough persistence is designed into the algorithm to keep it from leaving the CME decision state prematurely. As a consequence, the CME array is unavoidably contaminated with quasi-stationary flow, and this is reflected in the skewness of the $\mathrm{Mg} / \mathrm{O}$ distribution.

The second panel of Figure 2 shows the $\mathrm{He} / \mathrm{O}$ abundance ratio. As these are both high-FIP elements, little variation with speed or across regimes is expected or seen. A slight increase occurs between 250 and $425 \mathrm{~km} \mathrm{~s}^{-1}$, which may be attributed to the very high FIP of helium compared to oxygen [9]. The third panel shows $\mathrm{He} / \mathrm{H}$ abundance ratio $[\mathrm{He}]$ measured by Genesis. The values for the quasistationary wind range from about $3.4 \%$ to $4.1 \%$ with speed, slightly lower than previous surveys of [He] [11]. For the CME regime, the ratio is consistently higher, which is expected since an enhanced [He] value $(>7 \%)$ is one of the algorithm's possible criteria for CME selection. The [He] value in CMEs ranges from $4.4 \%$ to $8.3 \%$, increasing with speed, indicating that larger helium enhancements are generally found in faster CMEs.

We have also determined the oxygen charge state ratios as a function of velocity and for the different regimes. The oxygen charge state distribution in the solar wind is a measure of the electron temperature at the point in the corona where the distribution "freezes in". It is well established that the ratios of charge states, particularly for $\mathrm{O}^{7+} / \mathrm{O}^{6+}$, can be used to identify different coronal sources, in the same manner as elemental abundance ratios [4]. We see from the top panel of Figure 3 that the $\mathrm{O}^{7+} / \mathrm{O}^{6+}$ ratio distribution closely matches the $\mathrm{Mg} / \mathrm{O}$ ratio, even to the extent that there is a clear separation at intermediate speeds between the IS and $\mathrm{CH}$ flow regimes. This is a further indication that the regime algorithm is correctly differentiating between solar sources. In the bottom panel of Figure 3 we show the $\mathrm{O}^{8+} / \mathrm{O}_{\text {total }}$ ratio, where $\mathrm{O}_{\text {total }}$ is the total number of oxygen ions of all charge states. As we can see, the $\mathrm{O}^{8+}$ content is only about $\sim 2 \%$ in IS flow, and $<1 \%$ in $\mathrm{CH}$ flow, thus most often it is a negligible constituent. However, in CMEs, the $\mathrm{O}^{8+}$ content can extend beyond $10 \%$, and can contribute significantly to the concentrator collection sample. Thus, it is important to track all three charge states of oxygen in order to understand the distribution of oxygen on the concentrator targets.

\section{CONCLUSIONS}

By applying the Genesis algorithm results to ACE/SWICS abundance and charge-state data, we show that the solar wind speed history can be used to test the discrimination between the IS and $\mathrm{CH}$ flow types. By using a lower speed threshold for fast-toslow than for slow-to-fast regime transitions, the Genesis algorithm effectively compensates for effects that are due to transit to $1 \mathrm{AU}$. Furthermore, we show that on average, CME composition is independent of speed, having a composition signature most typical of the slow wind $<400 \mathrm{~km} \mathrm{~s}^{-1}$. However, differences between CMEs and the slow wind exist (see $\mathrm{He} / \mathrm{H}$ and $\mathrm{O}^{8+}$ ). The algorithm is successfully isolating the CME population, and thus protecting the $\mathrm{CH}$ and IS samples from contamination by CME material.

\section{REFERENCES}

1. D. S. Burnett, B. L. Barraclough, R. Bennett et al., Space Science Reviews, in press (2002).

2. J. E. Nordholt, R. C. Wiens, R. A. Abeyta et al., in press (2002).

3. J. Geiss, G. Gloeckler, and R. Von Steiger, Space Science Reviews 72, 49 (1995).

4. R. Von Steiger, N. A. Schwadron, L. A. Fisk et al., Journal of Geophysical Research 105, 27217 (2000).

5. B. L. Barraclough, E. E. Dors, R. A. Abeyta et al., Space Science Reviews, in press (2002).

6. M. Neugebauer, J. T. Steinberg, R. L. Tokar et al., Space Science Reviews, in press (2002).

7. G. Gloeckler, J. Cain, F. M. Ipavich et al., Space Science Reviews 86, 497 (1998).

8. R. von Steiger, R. F. Wimmer Schweingruber, J. Geiss et al., Advances in Space Research 15, 3 (1995).

9. J. Geiss, Space Science Reviews 33, 201 (1982).

10. R. von Steiger and J. Geiss, Advances in Space Research 13, 63 (1993).

11. W. C. Feldman, J. R. Asbridge, S. J. Bame et al., in The solar output and its variation, edited by $\mathrm{O}$. R. White (Colorado Associated University Press, Boulder, CO, 1977). 